\title{
Identification of the Model of the Dynamic System of a Milling Machine Based on the Study of Vibroacoustic Characteristics
}

\author{
Rustem Khusainov $^{1, *}$ and Dinar Safin ${ }^{1}$ \\ ${ }^{1}$ Kazan Federal University, Department of Design and Technology Software Engineering Industries, \\ 420008 Kazan Kremlyovskaya 18, Russian Federation
}

\begin{abstract}
In this paper, the authors propose a method for ensuring the reliability of the computational model intended for the study of dynamic processes in technological cutting systems. The advantage of using 3D models in $\mathrm{CAD} / \mathrm{CAM} / \mathrm{CAE}$ systems for solving such problems is shown. The method of conducting an experimental study to identify the calculated model by natural frequencies is justified. The method is proposed for adjusting the 3D model in order to ensure that the dynamic pattern occurring during cutting corresponds to experimental processes. The authors give a concrete example of solving the problem of identifying the computational model of the dynamic system of a milling machine. An example of solving the applied problem of optimizing cutting modes in terms of vibration resistance parameters using the identified computational model is also given.
\end{abstract}

\section{Introduction}

\subsection{The relevance}

When machining on metal cutting machines, it is always important to ensure vibration resistance. This is important both in terms of productivity and in terms of ensuring the quality of the machined surfaces. Traditionally, vibration resistance is provided empirically - by selecting cutting conditions, adjusting the technological system, etc. $[1,2]$. This increases production downtime associated with the implementation of such activities. On the other hand, digital production technologies are now spreading, which make it possible to perform computer simulation of the processes occurring during processing. [3, 4]. This allows directly at the stage of design and technological preparation of production to solve problems, including those associated with ensuring the vibration resistance of the technological system, and thereby sharply reduce downtime in the production process. However, to solve such problems, it is necessary to have a computer model that, with the required degree of reliability, would reflect the processes for the description of which it was

\footnotetext{
*Corresponding author: rmh@inbox.ru
} 
created. To do this, it is necessary to perform the identification [5] of such a model - to check the adequacy of the implemented processes, and, if necessary, to adjust it.

\subsection{State-of-the-art in modern mechanical engineering}

One of the most common methods for identifying a mathematical model of a dynamic system is the experimental measurement and analysis of vibroacoustic signals of the main units of the technological system. [6].

In the article [7], the authors concluded, that by registering vibroacoustic vibrations during cutting, one could then calculate the autocorrelation function, and then, after performing the appropriate mathematical processing according to the methods of the theory of automatic control, form the corresponding estimates correlated with the dynamic state of the technological system. The article [8] is devoted to obtaining a simple expression for determining the transfer function of a closed dynamic system of a lathe by an experimental and analytical method. The transfer function can be calculated if an analytical expression for the autocorrelation function of the output signal is obtained, for example, by identification by the experimentally recorded vibroacoustic vibrations of the cutter of a lathe. The article [9] is based on the analysis of the dynamic system identification method. A deterministic autonomous identification method has been proposed that can directly derive a reduced-order model from input and output data with a constant steady-state output, such as a transient function. The effectiveness of the method is demonstrated by numerical modeling.

Thus, to solve this problem, it is convenient to use the analysis of the amplitudefrequency characteristics of vibroacoustic signals, obtained during cutting on a machine tool. However, it is not necessary to use a special mathematical model to solve the problems of ensuring the vibration stability of processing.

\section{Dynamic analysis using finite element model}

One of the most well-known methods in this period for solving research problems to ensure the vibration stability of technological systems is finite element modeling and analysis in CAE systems [10, 11]. In this work, the Siemens NX system was used.

The modeling of the technological system during the analysis was carried out in the following sequence:

\subsection{Preparation of the 3D model of the technological system}

The composite model of a technological system is a hierarchical structure [12] that contains a number of key subassemblies consisting of several separate parts. As subassemblies were used:

- Units of the framework;

- Spindle assembly with bearings;

- Fixture;

- Tool assembly, including cutting and auxiliary tools [13, 14];

- Workpiece.

It is advantageous to prepare a 3D model in advance, since it is universal and can be used to solve various problems, for example, to simulate processing in a CAM system. [15].

For each element of the assembly, we set a set of physical properties. The material for each part is specified separately, which allows you to create accurate physical models of the investigated elements [16]. 


\subsection{Preparing of the finite element model}

At this stage, based on the created 3D model, a finite element mesh is formed for further calculation. It is efficient to construct a finite element model using the finite element assembly method, since the structure and relationships between the components of the model are preserved. After creating the finite element mesh for the entire assembly, one must assign physical constraint conditions that simulate the installation of the machine on the foundation and the connections between the components [17].

\subsection{Calculation of the natural frequencies of the dynamic system}

On the basis of the prepared finite element model, its solution was performed. In this work, the NX Nastran solver with solution type 103 "Real eigenvalues" was applied. As a result, the following values of natural frequencies were obtained: $251.201 \mathrm{~Hz} ; 513.413 \mathrm{~Hz} ; 571.67$ $\mathrm{Hz} ; 671.968 \mathrm{~Hz} ; 705.779 \mathrm{~Hz} ; 717.287 \mathrm{~Hz}$.

Figure 1 shows some of the natural modes. As can be seen from the figures, each natural frequency is determined by the properties of one component of the technological system.
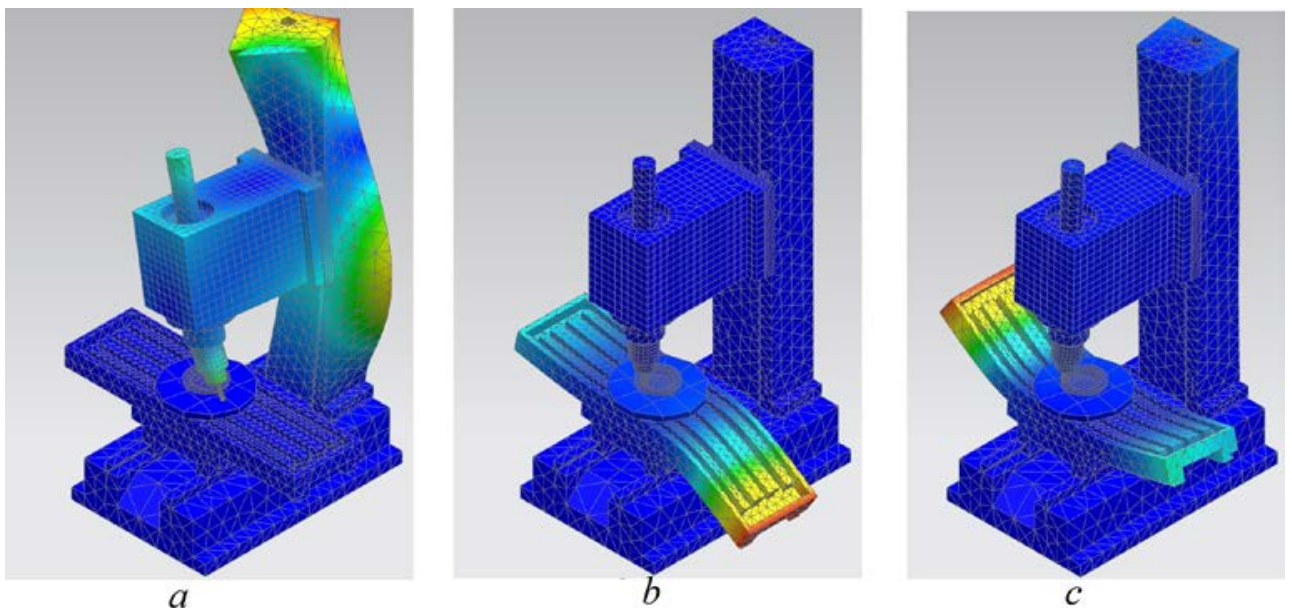

Fig. 1. a) natural mode 2, natural frequency - $513.413 \mathrm{~Hz}$; b) natural mode 3, natural frequency $571.67 \mathrm{~Hz}$; c) natural mode 6, natural frequency $-705.779 \mathrm{~Hz}$.

\section{Experimental determination of natural frequencies of the technological system}

Verification of the adequacy of the developed computational model consists in the fact that it is necessary to experimentally determine the natural frequencies of the technological system and check their compliance with the calculated frequencies. In this work, vibroacoustic analysis was used, which consists in analyzing the noise that occurs during the processing of the workpiece on the machine. The sources of the noise are mechanical vibrations, and the frequencies of acoustic signals correspond to the frequencies of mechanical vibrations. One of the most common methods of analyzing vibroacoustic signals is spectral analysis. The essence of the method is to decompose the signal into a set of harmonic oscillations and identify the frequencies of these oscillations. 
To determine the natural frequencies experimentally, a series of trials was carried out; in each, the workpiece was milled at different cutting speeds and with different feed rates. This allows us to excite resonant vibrations at different natural frequencies in each trial.

Six trials were carried out on shoulder milling with a cutting width of $1 \mathrm{~mm}$ and a depth of cut of $1 \mathrm{~mm}$ on a 45 steel workpiece with a $12 \mathrm{~mm}$ end mill with four teeth on a JDM$3 \mathrm{CNC}$ vertical milling machine. During the processing, sound was recorded using a portable microphone for subsequent analysis of the vibration frequencies that occur during the cutting process.

The values of the spindle speed and feed speed were selected so that in each trial the value of the frequency of the exciting force was close to one of the calculated frequencies. As the exciting frequency, we took the pulse repetition rate of the stepper motor of the feed drive:

$$
f_{p r}=\left(s_{\min } \cdot 4\right) /(t \cdot 60)
$$

where $f_{p r}$ - pulse repetition rate of the stepper motor of the feed drive, Hz; $s_{\min }$ - feed per minute, $\mathrm{mm} / \mathrm{min} ; \Delta$ - an increment; $t$ - pitch of the feed drive lead screw, $\mathrm{mm}$.

The cutting modes used for the experiment are shown in Table 1.

Table 1. Cutting modes in the experiment.

\begin{tabular}{|c|c|c|c|c|c|c|}
\hline № & $\begin{array}{c}\text { Cutting } \\
\text { depth, t, } \\
\mathbf{m m}\end{array}$ & $\begin{array}{c}\text { Cutting } \\
\text { width, B, } \\
\mathbf{m m}\end{array}$ & $\begin{array}{c}\text { Spindle } \\
\text { speed, n, } \\
\mathbf{r p m}\end{array}$ & $\begin{array}{c}\text { Feed per } \\
\text { tooth, sz, } \\
\mathbf{m m} / \mathbf{t o o t h}\end{array}$ & $\begin{array}{c}\text { Feed per } \\
\text { minute, } \\
\text { Smin, } \\
\mathbf{m m} / \mathbf{m i n}\end{array}$ & $\begin{array}{c}\text { Exciting } \\
\text { frequency, } \mathbf{f p r}, \\
\mathbf{H z}\end{array}$ \\
\hline 1 & 1 & 1 & 502 & 0,03 & 60,2 & 251 \\
\hline 2 & 1 & 1 & 1026 & 0,03 & 123,1 & 513 \\
\hline 3 & 1 & 1 & 1142 & 0,03 & 137 & 571 \\
\hline 4 & 1 & 1 & 1342 & 0,03 & 161 & 671 \\
\hline 5 & 1 & 1 & 1410 & 0,03 & 169,2 & 705 \\
\hline 6 & 1 & 1 & 1412 & 0,03 & 169,4 & 705,8 \\
\hline 7 & 1 & 1 & 1434 & 0,03 & 172 & 717 \\
\hline
\end{tabular}

After performing the spectral analysis, the amplitude-frequency characteristics of vibroacoustic signals were obtained in all experiments.

To assess the adequacy and reproducibility of the experiments, we used a comparison of variances according to Fisher's test, and as a verification method, we used the method of comparing the averages associated with the Student's criterion. The calculated values of the criteria turned out to be less than the table values; therefore, the conditions for the reproducibility of the experiments are fulfilled.

The frequencies at which the highest values of the signal amplitudes are observed were determined in all the frequency response. Based on the assumptions that the largest amplitudes arise in resonance modes, that is, when the natural frequencies coincide with the exciting frequencies, it can be concluded that the frequencies, at which the largest amplitude values are observed, are the natural frequencies.

Thus, the following experimental natural frequencies were identified (Table 2). As can be seen from the Table 2, the experimental frequencies are generally close to the calculated 
ones, but in some cases, there is a strong discrepancy. This can lead to errors when solving problems related to vibration resistance.

Table 2. The comparison of experimental and calculated natural frequencies.

\begin{tabular}{|c|c|c|c|c|c|c|}
\hline Calculated frequencies, $\mathrm{Hz}$ & 251 & 513 & 571 & 671 & 705,7 & 717 \\
\hline Experimental frequencies, $\mathrm{Hz}$ & 249,98 & 517,01 & 567,2 & 670,66 & 708,7 & 716,66 \\
\hline
\end{tabular}

\section{Adjustment of the calculation model}

Some natural frequencies obtained by the computational model and by the experimental one do not coincide. As can be seen from Table 2, the strongest discrepancies are observed in frequency pairs $513-517,01 \mathrm{~Hz} ; 571-567,2 \mathrm{~Hz} ; 705-708,7 \mathrm{~Hz}$. Therefore, it is necessary to adjust the calculated model so that the natural frequencies, calculated with its help, coincide with the experimental ones. This can be done by changing the properties of the finite elements that affect the frequencies.

The natural frequencies are affected by the mass and stiffness of the elements that determine this natural mode:

$$
f_{\mathrm{s}}(l, t) \sim \sqrt{ }(m / j)
$$

where $f_{s}$ - natural frequency; $m$ - element mass; $j$ - element stiffness.

The easiest way is to change the mechanical properties of materials, while both the stiffness characteristics and the mass characteristics change [18-20].

\subsection{Hz frequency tuning}

As can be seen from Fig. 1, this shape is determined by the torsional vibrations of the rack. Therefore, in order to bring the calculated frequency to the experimental frequency of $517.01 \mathrm{~Hz}$, that is, to increase the frequency, it is necessary to increase the rigidity of the rack, and it is precisely in torsion. Stiffness is set by the mechanical parameters of the material in the NX program. If we increase the thickness of the walls, this will entail a change in the frequency associated with the bending vibrations of the rack. There is no need to change this frequency. Therefore, we need to change some parameter in the material settings. From the mechanical properties of the material, the shear modulus affects torsional vibrations:

$$
G=\mu E
$$

where $\mu$ - Poisson's ratio, $E$ - Young's modulus, $G$ - shear modulus.

In order not to change the frequencies determined by the bending stiffness of the rack, it is necessary to change the Poisson's ratio. After changes and recalculations, the value of this coefficient was found, at which the value of this natural frequency reached $518.175 \mathrm{~Hz}$.

\section{2 $571 \mathrm{~Hz}$ and $705.7 \mathrm{~Hz}$ frequency tuning}

As can be seen from Fig. 1 a) and b), these shapes are determined by the bending vibrations of the table. The complexity of the task here is that the first frequency must be reduced, and the second one must be increased. As is known (2), the natural frequencies are influenced in different directions by the mass characteristics and the characteristics of the stiffness of the element. The first ones reduce the natural frequency, the second ones - increase. The density of the material determines the mass properties in NX. The bending vibrations of the 
table determine the frequency of $705.7 \mathrm{~Hz}$, so it is determined by the Young's modulus. Based on the foregoing, it was decided to reduce the frequency of $571 \mathrm{~Hz}$ by increasing the density of the material, and to increase the frequency of $705.7 \mathrm{~Hz}$ by increasing the Young's modulus. After simultaneous changes in these values and recalculations, frequencies of 569 $\mathrm{Hz}$ and $707.263 \mathrm{~Hz}$ were obtained, respectively.

Thus, as a result of changes in the characteristics of the materials of the components that determine the vibration modes at these frequencies, the calculated frequencies were brought to experimental values, which made it possible to adequately solve the problems of vibration stability using this finite element model.

\section{Applying the corrected model}

After adjusting the mechanical parameters of the materials of the components, the finite element model correctly simulates the dynamic behavior of the system under study, at least in the area of identifying resonance modes. Therefore, with the use of this model, it became possible to solve production problems in which vibration resistance is a limitation.

One of the production tasks is the selection of optimal cutting modes for roughing workpieces. Increasing productivity with higher cutting modes is hindered not only by the limitations of the machine drives and the limitations associated with the tool, but also by the problem of increasing vibrations when cutting modes are increased. One of the factors of increased vibration is the appearance of resonant vibrations when the cutting modes are incorrectly assigned. In order to exclude the occurrence of such processes in production conditions, it is necessary to check the vibration stability of the technological system during the design and technological preparation of production using the developed finite element model in the NX CAE program.

In the conditions of the study of the technological system considered above, it is necessary to choose the cutting modes so that there are no resonances with the natural frequencies of the considered system. The dynamic analysis of this technological system was carried out above.

These conditions correspond to the following cutting modes: cutting speed $24 \mathrm{~m} / \mathrm{min}$; milling speed $n=637 \mathrm{rpm}$; feed per tooth $0.03 \mathrm{~mm} /$ tooth; minute feed $76 \mathrm{~m} / \mathrm{min}$.

The experiment was performed under conditions similar to those described above. The calculated value of the Fisher test was 1.0412, the tabular value was 1.0414; the calculated value of the Student's test was 0.394, the tabular value was 1.282. Consequently, in this experiment, the reproducibility condition is satisfied.

According to the results of the experiment, the root-mean-square value of the vibroacoustic signal amplitude was $27.85 \mathrm{~dB}$. Table 3 shows the root-mean-square values of the spectrum amplitudes in the performed experiments:

Table 3. Root-mean-square values of amplitudes.

\begin{tabular}{|c|c|c|c|c|c|c|c|c|}
\hline № & 1 & 2 & 3 & 4 & 5 & 6 & 7 & 8 \\
\hline $\begin{array}{c}\text { Resonant } \\
\text { frequency, Hz }\end{array}$ & 249,98 & 517,01 & 567,2 & 670,66 & 708,7 & 708,7 & 716,66 & 316,7 \\
\hline $\begin{array}{c}\text { Root-mean-square } \\
\text { value of the } \\
\text { amplitude, dB }\end{array}$ & 75,8 & 53,73 & 50,1 & 58,6 & 49,8 & 61,3 & 39,6 & 27,85 \\
\hline $\begin{array}{c}\text { Cutting capacity, } \\
\text { mm 3min }\end{array}$ & 60,24 & 123,12 & 137,04 & 161,04 & 169,2 & 169,392 & 172,08 & 76 \\
\hline
\end{tabular}


According to the comparative results of the experiments from Table 3, it can be seen that in this experiment the root-mean-square amplitude is less than in the other experiments, therefore, the level of oscillations in experiment 8 is less than in the other experiments. Hence, it follows that the cutting conditions are chosen as optimal in terms of vibration resistance.

\section{Conclusion}

Thus, the main principles of this method are:

- Using a 3D model of a technological system in a CAD / CAM / CAE system, which can be used to solve other applied problems;

- Using the analysis of vibroacoustic signals generated during cutting in a technological system, which simplifies experimental research.

The use of this method is based on the following steps:

- Building a 3D model of a technological system;

- Dynamic analysis of the prepared model in the CAE system with the determination of natural frequencies;

- Conducting an experimental study consisting in performing short-term cuts on the machine tool, if it is possible with modes, that ensure the excitation of resonant modes at frequencies determined by calculation;

- Analysis of the experimental natural frequencies and their comparison with the calculated ones;

- Correction of the computational model in order to match the experimental natural frequencies.

This method is well integrated into the system of design and technological preparation of production using computer simulation, which allows you to minimize risks and downtime in the production process.

\section{References}

1. V.A. Kudinov, Machine dynamics (Mashinostroyeniye, Moscow, 1967)

2. Y.Y. Gorin, A.Y. Kryazhev, Y.Y. Tatarkin, Improving the vibration stability in end milling, Polzunovsky Vestnik, v. 2, pp. 43-48 (2015)

3. M. Iskandarov. R. Khisamutdinov. IOP Conf. Ser.: Mater. Sci. Eng. 570012033 (2019)

4. G. Bianchi, F. Paolucci, P. Van den Braembussche, H. Van Brussel, F. Jovane, CIRP Ann. - Man. Tech. 45 381-384 (1996)

5. R.R. Ziyatdinov, R.A. Biktimirov, IOP Conf. Ser.: Mater. Sci. Eng. 570012107 (2019)

6. M.P. Kozochkin, Rus. Eng. Res. 35 423-429 (2015)

7. A.A. Ignatiev, V.A. Karakozova, S.A. Ignatiev, Rus. Eng. Res. 35 123-125 (2015)

8. A.A. Ignatyev et al, IOP Conf. Ser.: Mater. Sci. Eng. 1047012005 (2021)

9. M. Kosaka, H. Uda, E. Bamba, H. Shibata, Dynamic system identification using a step input, J. of Low Freq. Noise Vib. and Act. Cont. 24 125-134 (2005)

10. D. Safarov, A. Kondrashov, Improving the efficiency of simulation of machine systems to perform simulation tasks, 2019 International Multi-Conference on Industrial Engineering and Modern Technologies (FarEastCon), pp. 1-5, (2019)

11. I.P. Balabanov et al, IOP Conf. Ser.: Mater. Sci. Eng. 915012005 (2020) 
12. D.T. Safarov, A.G. Kondrashov J. Phys.: Conf. Ser. 1546012041 (2020)

13. E.A. Ryabov, S.Y. Yurasov, O.I. Yurasova, Ball-end mill with a loxodromic cutting edge, Russian Engineering Research, v. 40, pp. 775-777 (2020)

14. E.A. Ryabov, S.Y. Yurasov, O.I. Yurasova, Producing the front surface in a ball-end mill, , Russian Engineering Research, v. 40, pp. 772-774 (2020)

15. A. Govorkov, A. Zhilyaev, The estimation technique of the airframe design for manufacturability, International conference on mechanical engineering, automation and control systems (MEACS2015), pp. 1757-8981 (2015)

16. M. Lavrentyieva, A. Govorkov, Identifying the objects in the structure of an e-model by means of identified formal parameters in the design and engineering environment, MATEC Web of Conferences, v. 129, p. 03002 (2017)

17. M. Law, Y. Altintas, A.S. Int. Jour. of Mach. Tools and Man. 68 81-90 (2013)

18. A.V. Shaparev, I.A. Savin, S.N. Ptichkin, Application of the polymeric material RIMAMID for production of machine parts, IOP Conf. Series: Materials Science and Engineering, v. 969, p. 012021 (2020)

19. R.V. Gavariev, I.A. Savin, E.N. Soldatkina, Choice of protective coating of metal molds for casting non-ferrous alloys, Solid State Phenomena, v. 299, pp. 867-871 (2020)

20. M. Lavrentyieva, A. Govorkov, Using a discrete product model to determine the design element junctures, MATEC Web of Conferences, v. 129, p. 03002 (2017) 